\title{
Activatable MRI probes for the specific detection of bacteria
}

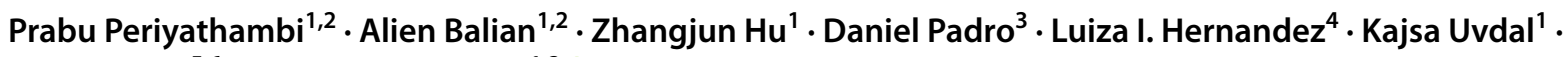 \\ Joao Duarte ${ }^{5,6} \cdot$ Frank J. Hernandez ${ }^{1,2}$ (1)
}

Received: 25 July 2021 / Revised: 7 September 2021 / Accepted: 30 September 2021 / Published online: 27 October 2021

(c) The Author(s) 2021, corrected publication 2022

\begin{abstract}
Activatable fluorescent probes have been successfully used as molecular tools for biomedical research in the last decades. Fluorescent probes allow the detection of molecular events, providing an extraordinary platform for protein and cellular research. Nevertheless, most of the fluorescent probes reported are susceptible to interferences from endogenous fluorescence (background signal) and limited tissue penetration is expected. These drawbacks prevent the use of fluorescent tracers in the clinical setting. To overcome the limitation of fluorescent probes, we and others have developed activatable magnetic resonance probes. Herein, we report for the first time, an oligonucleotide-based probe with the capability to detect bacteria using magnetic resonance imaging (MRI). The activatable MRI probe consists of a specific oligonucleotide that targets micrococcal nuclease (MN), a nuclease derived from Staphylococcus aureus. The oligonucleotide is flanked by a superparamagnetic iron oxide nanoparticle (SPION) at one end, and by a dendron functionalized with several gadolinium complexes as enhancers, at the other end. Therefore, only upon recognition of the MRI probe by the specific bacteria is the probe activated and the MRI signal can be detected. This approach may be widely applied to detect bacterial infections or other human conditions with the potential to be translated into the clinic as an activatable contrast agent.
\end{abstract}

Keywords MRI $\cdot$ Bacteria $\cdot$ Nucleic acid probes $\cdot$ Detection system $\cdot$ Nucleases $\cdot$ Activatable MRI contrast agents

\section{Introduction}

All types of activatable probes share a unique feature, they are turned "on" only after encountering the specific target or physical stimulus, while they remain "off" in the absence of such events [1,2]. Activatable probes have shown

Frank J. Hernandez

frank.hernandez@liu.se

1 Department of Physics, Chemistry and Biology, Linköping University, 58185 Linköping, Sweden

2 Wallenberg Centre for Molecular Medicine, Linköping University, Linköping, Sweden

3 Center for Cooperative Research in Biomaterials (CIC biomaGUNE), Basque Research and Technology Alliance (BRTA), 20014 Donostia-San Sebastián, Spain

4 Department of Clinical and Experimental Medicine, Linköping University, Linköping, Sweden

5 Department of Experimental Medical Science, Faculty of Medicine, Lund University, 22181 Lund, Sweden

6 Wallenberg Center for Molecular Medicine, Lund University, Lund, Sweden remarkable sensitivity and specificity because their target signal is maximized and the background signal is suppressed, facilitating a higher target-to-background ratio [3, 4]. Activatable probes can detect biological events at the nanoscale level (e.g., conformational changes or molecular recognition) using transduction mechanisms such as Förster resonance energy transfer (FRET) [5-8] and surface plasmon resonance (SPR) [9]. The best example in this regard is FRET where, upon excitement, a donor chromophore transfers its energy to an acceptor chromophore through non-radiative dipolar coupling. This energy transfer event is governed by one factor of the donor-acceptor distance [10]. Based on distance-dependent properties, activatable fluorescent probes allow the measurement of intracellular events, providing a powerful tool for studying fundamental aspects of cell biology and molecular recognition [11]. However, a major setback with this technology is the interference observed during the light-matter interactions, especially while dealing with in vivo biological systems like opaque tissues or organs embedded deep within the body [12]. Interestingly, some approaches based on enzymatic or smallmolecule probes have been reported for the identification of 
bacteria using fluorescence tracers $[13,14]$. In the last decades, activatable MRI contrast agents have been described [15-18], providing the opportunity to detect biochemical events at the cellular and molecular levels using MRI. We and others have previously shown a quenching effect on activatable MRI probes under different experimental conditions. Santra et al. [19] have encapsulated SPION and $\mathrm{Gd}^{3+}$ using $\mathrm{pH}$ polymeric materials, allowing the non-specific activation of the MRI probes by $\mathrm{pH}$ changes. We have then suggested that the distance between SPION and $\mathrm{Gd}^{3+}$ is a key parameter for constructing effective activatable MRI probes, using nucleic acids as linkers [20]. Much later, Choi et al. have confirmed our initial findings by measuring the critical distance needed to obtain activatable MRI probes [21, 22]. Activatable MRI probes can overcome the limitation of fluorescence-based systems, with a mature MRI technology that offers the advantages of unlimited tissue penetration and high spatial resolution, which are exceptional features for clinical approaches [23].

In this study, we developed an activatable MRI probe with a "turn-on" switch for detecting a specific nuclease, micrococcal nuclease (MN) secreted by Staphylococcus aureus ( $S$. aureus). S. aureus is a major cause of bacterial infections in humans and its virulence is characterized by high morbidity and mortality, as in the notorious case of methicillin-resistant S. aureus (MRSA) infections [24]. More generally, $S$. aureus is behind severe and difficult to diagnose conditions that may implicate resistant or non-resistant strains, such as osteomyelitis, endocarditis, infantile pneumonia, or septic arthritis, with significant clinical and economic impact [25]. For these reasons, the prevention and control of $S$. aureus has been identified as a public health priority. In order to prevent and control S. aureus-related infections, rapid and accurate diagnostic methods are essential for the timely and appropriate treatment, especially in the case of MRSA.

In our study, we use nucleases as biomarkers. Nucleases are proteins with tremendous diversity and widespread expression that could be used to specifically identify a variety of bacterial species [26]. Besides their detection by immune assays or by molecular techniques, as degrading enzymes, nucleases can be detected by their activity towards/ degradability of oligonucleotide substrates. This activity can be therefore detected by activatable oligonucleotide probes that can be further incorporated in different types of diagnostic biosensors. Herein, we report on the development of activatable MRI probes that detect bacteria and work as a contrast agent. In a previous proof-of-concept study, a molecular imaging approach that rapidly and specifically detects $S$. aureus infections was reported. This activatable fluorescent probe, the sequence of which is referred to as TT probe, is turned "on" by micrococcal nuclease (MN) specifically secreted by these bacteria [27]. With this strategy, we have developed an oligonucleotide probe with high sensitivity and specificity that allow in vivo detection of bacteria in $45 \mathrm{~min}$, which clearly demonstrates the potential of this technology for targeting applications where nuclease activity could be detected. We have previously demonstrated the specificity of TT probe to MN secreting S. aureus among other strains and other bacteria in addition to the resistance of the probe in human and mouse serum [27]. Based on this knowledge and as a proof of concept study, we have designed an activatable MRI probe composed of a core superparamagnetic iron oxide nanoparticle (SPION) bearing $S$. aureus-specific short oligonucleotide sequences that are linked to dendrons functionalized with several gadolinium ion $\left(\mathrm{Gd}^{3+}\right)$ complexes. Incorporation of dendrons in MRI contrast agents and the resulted benefits such as the enhanced relaxivity have been widely described [28-31]. In the MRI probe described in this paper, the short distance between SPION and $\mathrm{Gd}^{3+}$ induces a quenching effect of SPION to the magnetic relaxation $\left(T_{1}\right.$-based) of $\mathrm{Gd}^{3+}$, and this effect is described as "magnetic quenching." Then, in the presence of the nucleases associated to $S$. aureus bacteria, the oligonucleotides are specifically cleaved, thus releasing $\mathrm{Gd}^{3+}$ complexes from the superparamagnetic SPION core, resulting in "on" $T_{1}$ relaxation. Figure 1 depicts the schematic structure and

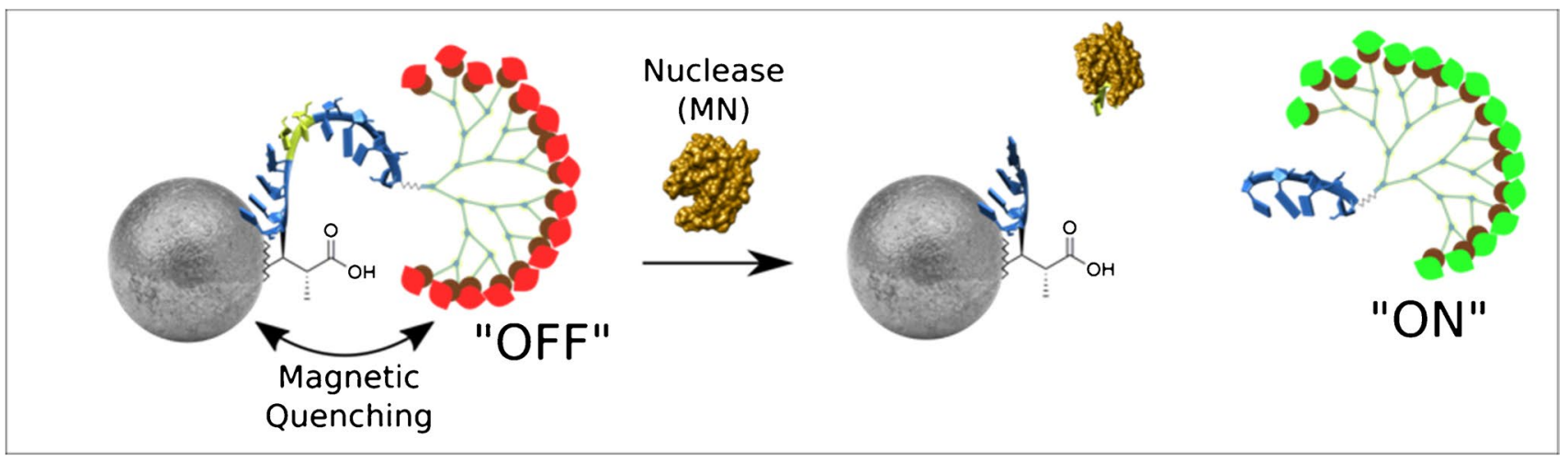

Fig. 1 Activation of the MRI probe by specific nuclease activity derived from bacteria 
mechanism of activatable MRI probes for detecting specific bacteria using nucleases as biomarkers and oligonucleotides as recognition molecules. To the best of our knowledge, this is the first approach that detects bacteria-derived protein with an activatable MRI probe using SPIONs and dendrons functionalized with $\mathrm{Gd}^{3+}$. This work reports an efficient activatable MRI probe that exhibits high sensitivity due to high signal-to-background ratios and with unlimited tissue penetration provided by the MRI signal acquisition; these features facilitate the translation of this approach into the clinical settings.

\section{Material and methods}

\section{Materials}

Oleic acid-coated iron oxide nanoparticles (20-nm SPION) dispersed in chloroform were obtained from Ocean NanoTech (San Diego, CA). $\mathrm{GdCl}_{3}$, meso-2,3-dimercaptosuccinic acid (DMSA), tryptic soy agar (TSA), and tryptic soy broth (TSB) were purchased from Sigma-Aldrich (St. Louis, MO, USA). Sulfosuccinimidyl 4-(N-maleimidomethyl) cyclohexane-1-carboxylate (Sulfo-SMCC), sodium L-ascorbate, and copper (II) sulfate were purchased from Thermo Scientific, Uppsala, Sweden. bis-MPA ammonium dendron, acetylene core, Generation 4 (G4) were obtained from Polymer Factory, Sweden AB. $p$-SCN-Bn-DTPA was obtained from Macrocyclics, USA. Tris [3-hydroxypropyltriazolylmethyl] amine (THPTA) was purchased from Click Chemistry Tools LLC. PD MiniTrapTM G-10 was purchased from GE Healthcare. MiniMACS separator and MACS MS column were purchased from Miltenyi Biotec (cat\#130-042-102). All other chemicals and reagents were of reagent grade and used without further purification.

\section{Methods}

\section{Preparation of the DMSA-coated SPION (D-SPION)}

Ligand exchange was performed to exchange capping molecule from oleic acid (organic phase) to DMSA (water-soluble phase) on surface of 20-nm iron oxide nanoparticles (SPION). Two milligrams of 20 -nm SPION in $500 \mu \mathrm{L}$ of toluene and DMSA [ $5 \mathrm{mg}$ ] in $500 \mu \mathrm{L}$ of methanol were mixed and shaken (1000 rpm) for $12 \mathrm{~h}$ at room temperature (RT) until two phases appear. After incubation, the nanoparticles were centrifuged at $3000 \mathrm{rpm}$ for $10 \mathrm{~min}$. The final black solid was air dried and redispersed in distilled water. The $\mathrm{pH}$ of the resulted solution was adjusted to 10 . The unreacted DMSA was removed via MACS MS column.
Labelling of TT probe on D-SPION (D-SPION-TT-N ${ }_{3}$ )

A total of $150 \mu \mathrm{L}$ of Sulfo-SMCC $(5 \mathrm{mM})$ was dissolved into $100 \mathrm{nmol}(10 \mu \mathrm{L})$ of TT probe $\left(5^{\prime}-\mathrm{NH}_{2}\right.$-mC-mU-mC-mG-TT-mC-mG-mU-mU-mC-N ${ }_{3}-3^{\prime}$ ) and the mixture was shaken at $1500 \mathrm{rpm}$ for $1 \mathrm{~h}$ at RT. Next, $0.25 \mathrm{~mL}$ of D-SPION was added and the mixture was shaken at $500 \mathrm{rpm}$ for an additional $2 \mathrm{~h}$ at RT [32]. D-SPION@TT-N 3 probe was then transferred to $500 \mu \mathrm{L}$ of sodium bicarbonate buffer $\mathrm{pH} 8.8$ using a MACS MS column.

\section{Click chemistry CuAAC reaction (D-SPION@TT-G 4 )}

To carry out the reaction, first, $500 \mu \mathrm{L}$ of D-SPION@TT-N 3 was mixed with $100 \mu \mathrm{L}$ of $\mathrm{G} 4$ dendron alkyne $(0.06 \mathrm{mmol})$ prepared in DMF. Next, $50 \mu \mathrm{L}$ of $500 \mathrm{mM}$ THPTA/100 mM $\mathrm{CuSO}_{4}$ and $25 \mu \mathrm{L}$ of sodium L-ascorbate $(50 \mu \mathrm{mol})$ were added to the reaction mixture. After $3 \mathrm{~h}$ of incubation, the nanoparticles were purified on a MACS MS column with sodium bicarbonate buffer, pH 8.8. Then, the D-SPION@ TT- $\mathrm{G}_{4}$ probe was transferred to $500 \mu \mathrm{L}$ of sodium bicarbonate buffer at $\mathrm{pH} 8.8$ by MACS MS column [33].

\section{Preparation of the activatable MRI probe}

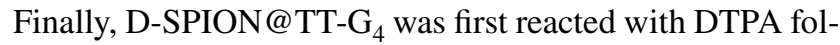
lowed by $\mathrm{Gd}^{3+}$ chelation using gadolinium chloride. The reaction was performed as follows: $300 \mu \mathrm{L}$ of D-SPION@ TT- $\mathrm{G}_{4}$ was mixed with $500 \mu \mathrm{L}$ of $p$-SCN-DTPA ( $5 \mathrm{mg}$; sodium bicarbonate buffer $\mathrm{pH} 8.8$ ) at $\mathrm{RT}$, overnight. The $\mathrm{pH}$ of the reaction mixture was adjusted to $8 \sim 9$ by adding $1 \mathrm{~N} \mathrm{NaOH}$ and MACS MS column was used to purify

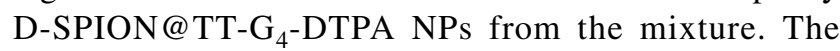
D-SPION@TT-G 4 -DTPA was transferred to water $(\mathrm{pH}$ adjusted to 7.4) to a final volume of $500 \mu \mathrm{L}$. In total, 200 $\mu \mathrm{L} \mathrm{GdCl}{ }_{3}$ solution $(2.64 \mathrm{mg})$ was added to $300 \mu \mathrm{L}$ of

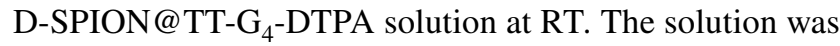
adjusted to $\mathrm{pH} 8$ and stirred overnight. The resulting probe

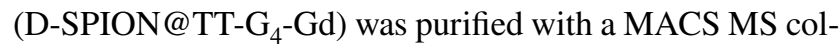
umn to a final volume of $250 \mu \mathrm{L}$ aqueous solution.

\section{Activatable MRI probe characterization}

Transmission electron microscopy (TEM) The size and shape of the activatable MRI probe were analyzed using TEM (with a FEI Tecnai G2 operated at $200 \mathrm{kV}$ ). A total of $10 \mu \mathrm{L}$ of probe dispersed in an aqueous solution $\left(2 \mathrm{mg} \mathrm{mL}^{-1}\right)$ was deposited directly on an amorphous carbon film supported copper TEM grid.

Dynamic light scattering (DLS) The hydrodynamic diameters of D-SPION and MRI activatable probe were measured using a Zetasizer Nano ZS90 (Malvern Instruments Ltd., 
Worcestershire, UK). Dispersion D-SPION and activatable MRI probe were prepared at a concentration of $0.1 \mathrm{mg} \mathrm{mL}^{-1}$ in deionized water, ultrasonicated for $60 \mathrm{~min}$. Next, the solution was filtered with a $0.22-\mu \mathrm{m}$ filter and finally subjected to analysis.

UV-Vis spectroscopy PerkinElmer Lambda 950 UVVis spectrometer was used to record the absorbance of D-SPION, TT oligonucleotide probe, and the activatable MRI probe. The samples were diluted in deionized water $\left(0.1 \mathrm{mg} \mathrm{mL}^{-1}\right)$ and the spectra were recorded at the wavelength of $200-800 \mathrm{~nm}$.

Magnetic quenching evaluation Preparation of G4-dendron$\mathrm{Gd}^{3+}$ complex was obtained by conjugating a solution of G4-dendron- $\mathrm{NH}_{2}(1.0 \mathrm{mg}, 0.043 \mathrm{mmol})$ with p-SCN-Bn DTPA $(5 \mathrm{mg}, 0.39 \mathrm{mmol})$ in $1 \mathrm{~mL}(0.1 \mathrm{M}$ sodium bicarbonate buffer) at RT. The solution was stirred for $12 \mathrm{~h}$, then filtered 3 times using an Amicon Ultra-15 (3000 MWCO) column at $4500 \mathrm{~g}$ for $5 \mathrm{~min}$. $\mathrm{G}_{4}$-Dendron-DTPA $(10,20$, 30,40 , and $50 \mu \mathrm{g}$ ) was added to different concentrations of $0.04,0.08,0.12,0.16$, and $0.2 \mathrm{mM} \mathrm{GdCl}_{3} \cdot 6 \mathrm{H}_{2} \mathrm{O}$ in $220 \mu \mathrm{L}$ for $2 \mathrm{~h}$ at $40^{\circ} \mathrm{C}$, respectively. Then, all samples were loaded in 7.5-mm-diameter NMR tubes and analyzed with a Bruker minispec mq60 NMR analyzer at $37{ }^{\circ} \mathrm{C}$ using a magnetic field of $1.41 \mathrm{~T}$ (inversion time (IT) $=50,100,200,300,500$, 1000,2000 , and $3000 \mathrm{~ms}$ ). Each sample was temperaturestabilized for $4 \mathrm{~min}$ before measurement. Then, $r_{1}$ values calculated from the slope of $1 / T_{1}$ graph were plotted against the $\mathrm{Gd}^{3+}$ concentration and compared with the activatable MRI probe under the same conditions.

Inductively coupled plasma mass spectroscopy The quantification of Fe and Gd was performed using ICP-MS by ALS Scandinavia AB (Sweden).

\section{Preparation of bacterial supernatant}

Staphylococcus aureus (ATCC 29,213) and Staphylococcus epidermidis (ATCC 39,584) were cultivated in respective petri dishes with tryptic soy agar (TSA) medium supplemented with defibrinated sheep blood (Thermo Scientific, Inc., Waltham, MA, USA). Growth of individual bacterial colonies was allowed using the quadrant method by streaking a porous glass bead directly in the petri dishes. Following 24-h incubation at $37{ }^{\circ} \mathrm{C}$, a single colony was transferred from solid media into $50 \mathrm{~mL}$ tryptic soy broth (TSB) and incubated for $24 \mathrm{~h}$ at $37^{\circ} \mathrm{C}$ and $200 \mathrm{rpm}$. The liquid cultures were diluted (1:500) in TSB and incubated for $24 \mathrm{~h}$ at $37^{\circ} \mathrm{C}$ and $200 \mathrm{rpm}$. The bacterial culture is estimated to be in the stationary phase with values greater than $10^{9} \mathrm{CFU} /$ $\mathrm{mL}$ after this incubation time of $24 \mathrm{~h}$. Next, the culture was centrifuged at $4500 \times g$ for $30 \mathrm{~min}$ and supernatants were collected for direct use or storage at $4{ }^{\circ} \mathrm{C}$ or $-20{ }^{\circ} \mathrm{C}$. TSB culture medium was used as control [34]. Bacterial counts were calculated using McFarland standards and reported as colony forming units (CFU) per milliliter.

\section{Relaxation $T_{1}$ measurement using NMR Minispec (1.41 T)}

The activatable MRI probes at different $\mathrm{Fe}$ concentrations $(0.005,0.01,0.02,0.03$, and $0.04 \mathrm{mM})$ were incubated with $S$. aureus (targeting bacteria) and S. epidermidis (nontargeting bacteria) cultivated media or TSB. All reaction mixtures were incubated at $37{ }^{\circ} \mathrm{C}$ for $1 \mathrm{~h}$ and loaded into 7.5-mm-diameter NMR tubes and measured with a Bruker minispec mq60 NMR analyzer at $37^{\circ} \mathrm{C}$ using a magnetic field of $1.41 \mathrm{~T}$ (IT) $=50,100,200,300,500,1000,2000$, and $3000 \mathrm{~ms}$. Each sample was temperature stabilized for 4 min before measurement. Then, $r_{1}$ values calculated from the slope of the $1 / T_{1}$ graph were plotted against the $\mathrm{Fe}$ concentration.

\section{MR imaging measurements}

The MRI nanoprobe was incubated for $1 \mathrm{~h}$ with culture media, S. epidermidis (non-targeting bacteria) and S. aureus (targeting bacteria). Then, the probe was loaded into Wilmad NMR tubes 3-mm diameter at concentrations of 0.03 and $0.07 \mathrm{mM} \mathrm{Fe}$ and incubated at $37^{\circ} \mathrm{C}$ during MRI scan. MRI scans were carried out in a preclinical 9.4-T magnet (Agilent, Palo Alto, CA, USA) interfaced to Avance III electronics, using a quadrature transmit-receive coil (Bruker, Ettlingen, Germany). $T_{1}$ values were estimated from images acquired using the rapid acquisition with relaxation enhancement (RARE) sequence with inversion recovery (IT $=50,200$, $400,800,1500,3000,5500,8000,12,000 \mathrm{~ms}, \mathrm{TE}=7.0 \mathrm{~ms}$, echo train length 2 , data matrix size $128 \times 64$, field of view $30 \times 15 \mathrm{~mm}^{2}$, slice thickness $=3 \mathrm{~mm}, 1 \mathrm{scan}$ ).

\section{Cell culture and cytotoxicity assay}

The CCD18LU fibroblasts obtained from American Type Culture Collection (ATCC) (Manassas, VA, USA) were seeded in 96-well clear bottom plates, at the density of $1 \times 10^{4}$ cells/well. The cells were maintained in minimal essential medium (MEM) supplemented with fetal bovine serum (FBS), L-glutamine, and penicillin/streptomycin. All cell culture reagents were purchased from Gibco (Thermo Fisher Scientific, USA). After 24 -h incubation at $37^{\circ} \mathrm{C}$, the cells were treated for 24 or $48 \mathrm{~h}$ with $100 \mu \mathrm{L}$ cell culture medium containing MRI probe at $0.07 \mathrm{mM}$ Fe. Untreated cells were supplemented with fresh medium only. Next, nucleic acid stain (CyQUANT Direct Cell Proliferation Assay Kit, Invitrogen, UK) was added at a volume of $100 \mu \mathrm{L} /$ 
well. After 1-h incubation at $37{ }^{\circ} \mathrm{C}$, bright field and fluorescence microscopy images were acquired using Cytation 1 (Cell Imaging Multi-Mode Reader, BioTek, USA), and Gen5 software with $\mathrm{a} \times 4$ optical objective was used for cell viability counts.

\section{Results}

We and others have reported that $T_{1}$ relaxivity of $\mathrm{Gd}^{3+}$ can be quenched by superparamagnetic (SPION) in the local magnetic field [35]. The high transverse relaxivity $\left(r_{2}\right)$ effect of SPION serves as a quencher to control the longitudinal relaxivity $\left(r_{1}\right)$, and practically negligible $T_{1}$ contrast effects for activatable MRI probes are observed [33]. Figure 2 shows the schematic synthesis route of the activatable MRI probes used in this study. The synthesis begins with SPIONs of 20-nm diameter that were modified with dimercaptosuccinic acid (DMSA). This step converts the hydrophilic nanoparticles into hydrophilic SPIONs with coupling groups for further conjugation (D-SPION). An oligonucleotide (TT probe) that specifically recognizes nucleases derived from S. aureus was used as a biorecognition molecule [36].

The oligonucleotide TT probe was synthesized with amine and azide coupling groups at 5' and 3 '-end, respectively, for further conjugation. Finally, the activatable MRI probe was assembled by flanking the oligonucleotide TT probe with D-SPION at the 5 '-end and by the dendron functionalized with gadolinium complex at the 3 '-end. A detailed description of the synthesis route is provided in the
"Methods" subsection. To confirm the amounts of Fe and $\mathrm{Gd}^{3+}$ bared by the activatable MRI probes, we quantified the elemental composition by inductively coupled plasma mass spectroscopy (ICP-MS) analysis. The ICP-MS analysis determined concentrations of 8,23 , and $270 \mathrm{mg} / \mathrm{kg}$ for $\mathrm{Fe}$ and $\mathrm{Gd}^{3+}$, respectively. These results suggest an efficient functionalization of $\mathrm{Gd}^{3+}$ complexes over the D-SPION surface, and a successful methodology to obtain activatable MRI probes.

Next, microscopy features such as size and monodispersity of D-SPIONs and activatable MRI probes were evaluated by transmission electron microscopy (TEM). Figure 3A shows D-SPION hydrophilic nanoparticles with an ellipsoidal shape with an approximate size of $20 \mathrm{~nm}$. Interestingly, Fig. 3B shows an increase in size, up to $24 \mathrm{~nm}$, for the activatable MRI probes. This $\sim 4-\mathrm{nm}$ increase in size is consistent with the oligonucleotide and dendron conjugation that can be observed as an interstitial space (Fig. 3B, arrows) between the D-SPION and the solvation layer decorated with $\mathrm{Gd}^{3+}$. These results are in good agreement with previous TEM images of similar MRI probe constructs [37]. Next, the colloidal stability in water of the D-SPION and activatable MRI probes has been evaluated by DLS. Figure $3 \mathrm{C}$ shows the mean hydrodynamic size of $23.26 \mathrm{~nm}$ for D-SPION, which is slightly larger when compared with the $20 \mathrm{~nm}$ reported by TEM analysis. The activatable MRI probe construct has reported an average hydrodynamic size of $34.85 \mathrm{~nm}$. These results are consistent with the conjugation of oligonucleotide and dendron thickness that form the solvation layer observed by TEM (Fig. 3B). Altogether, TEM and DLS

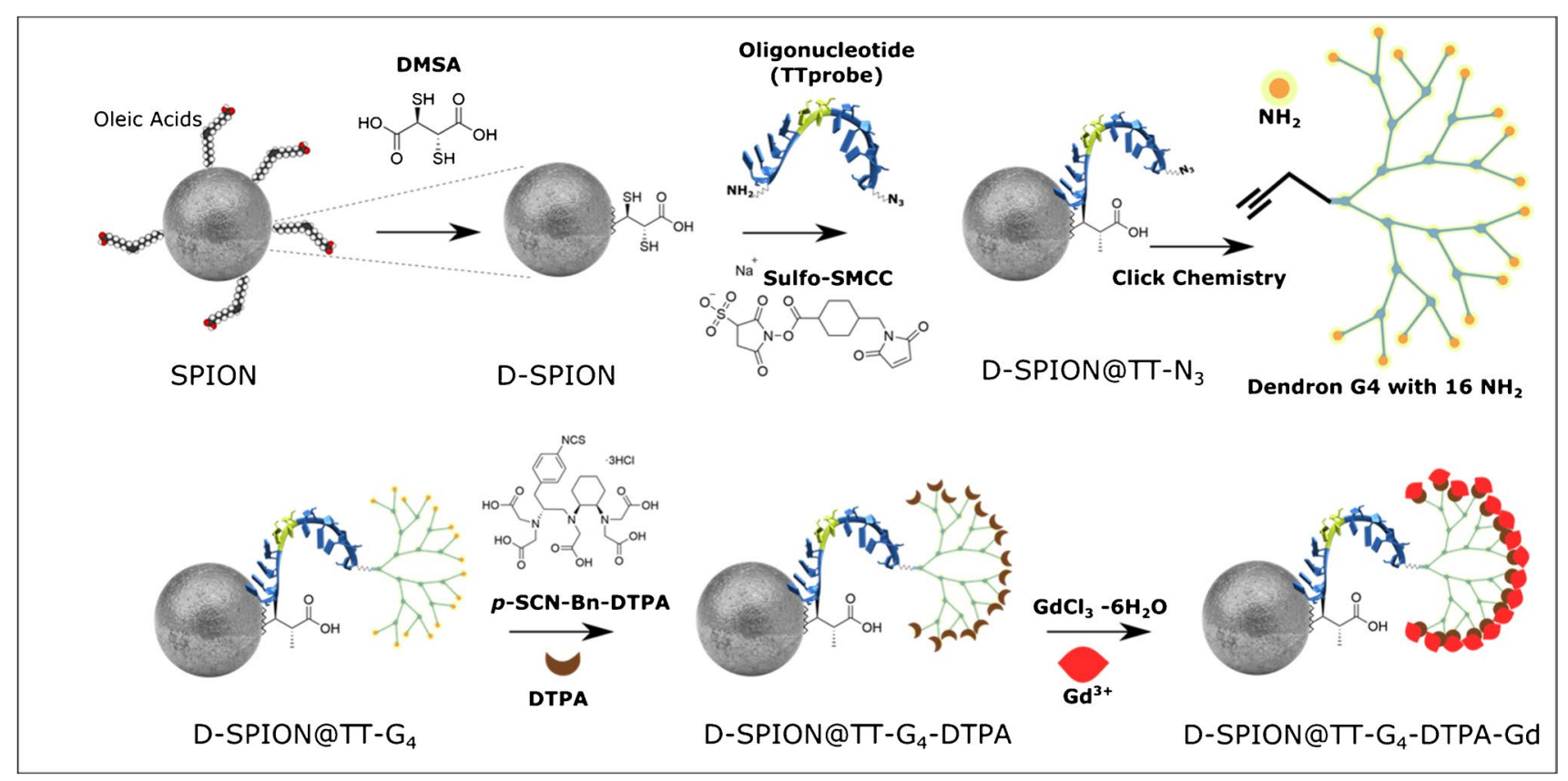

Fig. 2 Schematic of the activatable MRI probe synthesis 
Fig. 3 Characterization of activatable MRI probes. TEM images were acquired for (A) D-SPION and (B) activatable MRI probe; the arrows show the solvation layer between D-SPION and $\mathrm{Gd}^{3+}$. (C) The hydrodynamic sizes of D-SPION and activatable MRI probe were obtained by DLS analysis. (D) The UV-Vis profiles of D-SPION, oligonucleotide TT probe, and activatable MRI probe were obtained by $\mathrm{UV}-\mathrm{Vis}$ spectroscopy analysis
A
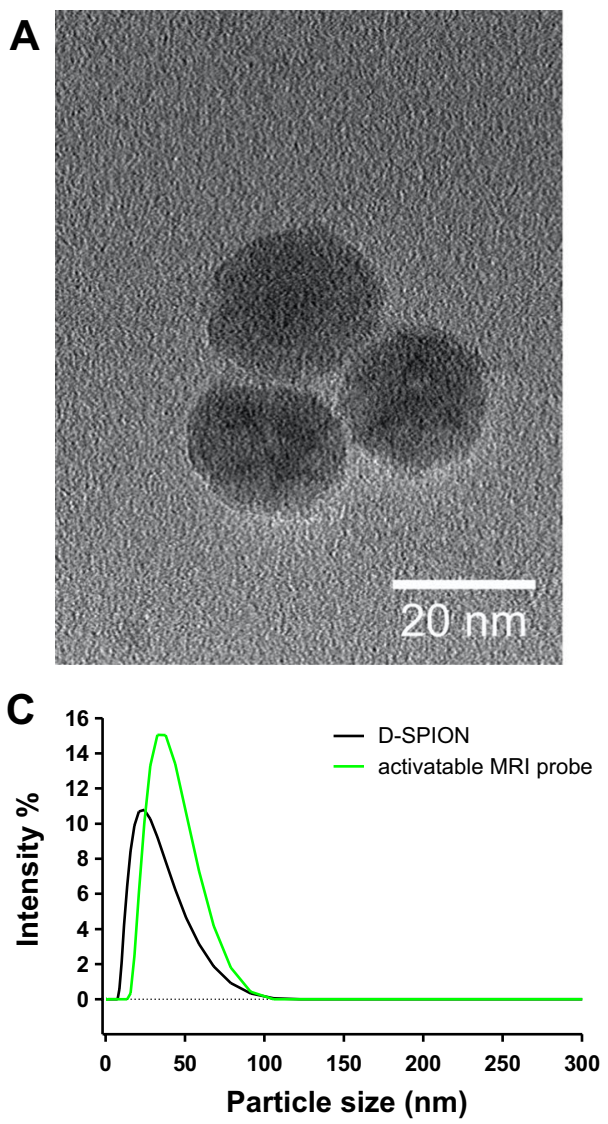

B
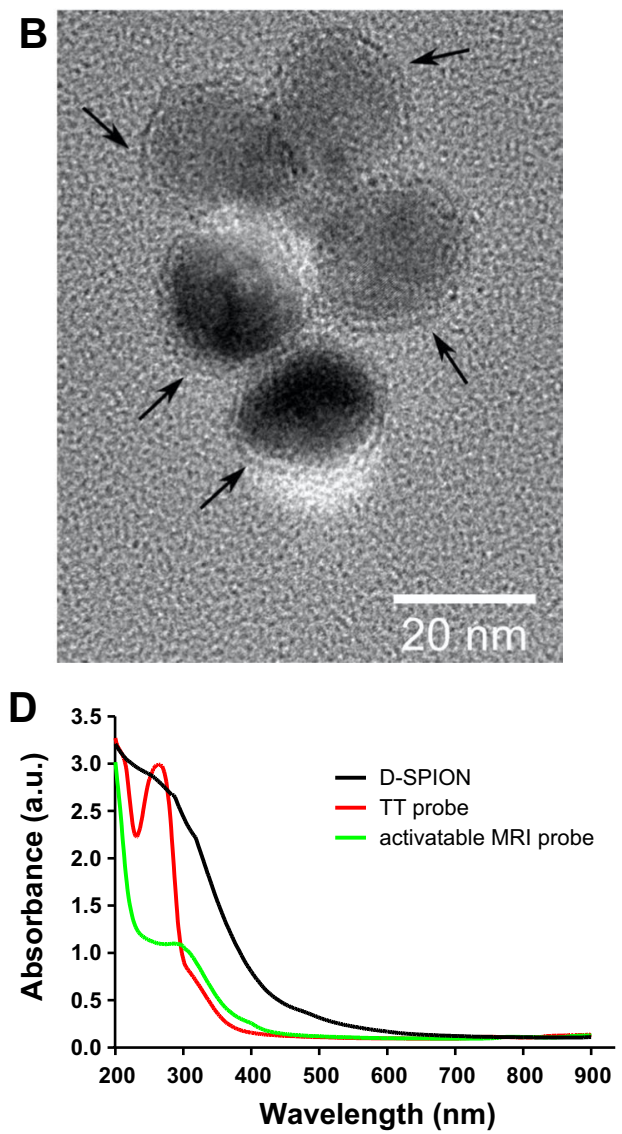

results confirmed that no aggregation occurs after the synthesis of the activatable MRI probes and hydrodynamic size distributions suggest a monodisperse colloidal system. To complete the characterization of the activatable MRI probes, UV-Vis absorption spectroscopy was performed to confirm the incorporation of the oligonucleotide during the synthesis process. These measurements were carried out in water at room temperature. Figure 3D shows the individual UV-Vis profile of D-SPION (black line), where no clear peak was observed. In contrast, the UV-Vis profile of the oligonucleotide (TT probe) has shown a clear peak at $264 \mathrm{~nm}$ that is consistent with the chemically modified oligonucleotides (red line). Furthermore, the activatable MRI probe profile (green line) has exhibited a broad peak from 285 to $340 \mathrm{~nm}$ indicating the presence of the oligonucleotide in the MRI construct. These results confirm the successful synthesis of the activatable MRI probes, and more specifically the incorporation of the oligonucleotide into the MRI probes.

Once the activatable MRI probes were synthesized and fully characterized, we tested their functionality as contrast agents. To demonstrate the functionality of the activatable MRI probes, first we calculated the "magnetic quenching" obtained after synthesis. We therefore compared the $T_{1}$ relaxivity signals of dendron $\mathrm{G} 4$ functionalized with $\mathrm{Gd}^{3+}$ (Dendron-Gd ${ }^{3+}$ complex) and the activatable MRI probes.
Dendron-Gd ${ }^{3+}$ complexes (Fig S1, squares) have reported higher $T_{1}$ relaxivity signal of $46.018 \mathrm{mM}^{-1} \mathrm{~s}^{-1}\left(\mathrm{r}_{1}\right)$, led by prolonged rotational correlation time $(\tau R)$. In contrast, the activatable MRI probe with the $\mathrm{Gd}^{3+}$ quenched by the close proximity with D-SPION (Fig S1, circles) has shown a lower $T_{1}$ relaxivity signal of $12.586 \mathrm{mM}^{-1} \mathrm{~s}^{-1}$, resulting in a $T_{1}$ signal difference of 3.66 -fold. These results indicate that the "magnetic quenching" effect was obtained after synthesis and that the activatable MRI probes are ready to be tested using biological samples. Furthermore, we have tested the cytotoxicity of the MRI probe by a fluorescencebased cell viability assay. The results of cell viability counts have shown that cells were not significantly affected after incubating the MRI probe $(0.07 \mathrm{mM} \mathrm{Fe})$ for 24 and $48 \mathrm{~h}$ (Fig S2). Next, we sought to evaluate whether the activatable MRI probes have the capability to identify bacteria in a specific manner, in standard bacterial cultures after $24 \mathrm{~h}$. Thus, the activatable MRI probes were incubated for $1 \mathrm{~h}$ at $37{ }^{\circ} \mathrm{C}$ with bacterial cultures of $S$. aureus as the target bacteria $\left(2.4 \times 10^{9} \mathrm{CFU} / \mathrm{mL}\right)$ and $S$. epidermidis as the control bacteria $\left(2.1 \times 10^{9} \mathrm{CFU} / \mathrm{mL}\right)$, along with bacterial culture media as background control. Importantly, S. epidermidis does not produce $\mathrm{MN}$, the nuclease needed to activate the MRI probes. Then, the $T_{1}$ relaxivities of $\mathrm{Gd}^{3+}$ derived from the MRI probes were measured at $60 \mathrm{MHz}$ using an NMR 
MiniSpec (Fig. 4). The MiniSpec device allows the acquisition of rapid and simple relaxivity measurements that facilitate the optimization of the activatable MRI probes. The relaxivity results have shown that $S$. epidermidis and bacterial culture media did not show a significant change at several concentrations of the activable MRI probes. In contrast, a clear shift was observed when the MRI probes were incubated with $S$. aureus, showing higher relaxivity signals than both controls. Moreover, a concentration-dependent increase in relaxivity signal was only observed for $S$. aureus samples. These results confirm the specific recognition of a bacterium using an activatable MRI probe and the capability of this approach to add diagnostic value to the MRI-based systems by incorporating a novel type of contrast agent.

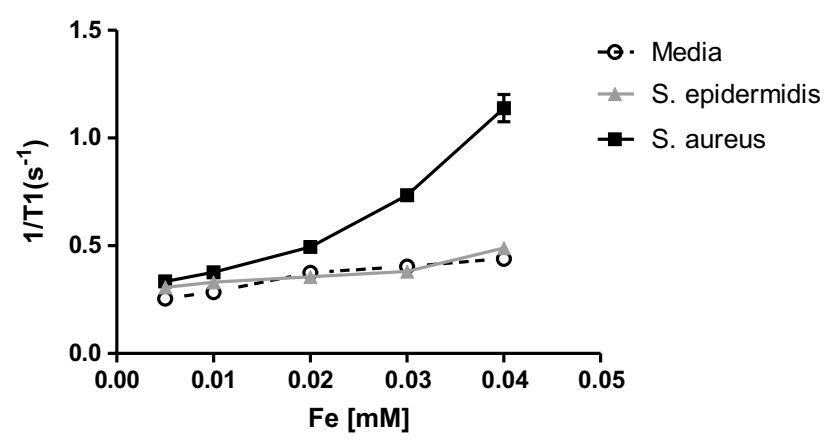

Fig. 4 Activatable MRI probe performance. (A) Schematic mechanism of action of the activatable MRI probes. (B) Specific detection of bacteria using activatable MRI probes and data acquisition by Minispec $1.4 \mathrm{~T}$
To demonstrate the potential applicability in the clinic of this type of activatable MRI probes, we have repeated the detection of specific bacteria using an MRI machine (Bruker BioSpec Avance III). For the MRI analysis, we used the same samples and experimental conditions described in the previous relaxivity studies. Figure 5A shows the $T_{1}$ relaxation times acquired by the MRI machine. The samples containing $S$. aureus have shown lower relaxation times compared with both controls, nontargeted bacteria and culture media, suggesting that this activatable strategy is an effective manner of identifying bacteria using an MRI system. Furthermore, we calculated the relaxation rate $1\left(R_{1}\right)$ as the inverse of relaxation time $\left(1 / T_{1}\right.$ relaxivity $\left.\mathrm{S}^{-1}\right)$. Figure $5 \mathrm{~B}$ shows the $R_{1}$ calculations, and any value over 1 (arbitrary threshold) is considered positive for $S$. aureus. Consistent with the previous results, the MR images acquired with an 9.4-T magnet have shown an increase in intensity for $\mathrm{Gd}^{3+}$ when the activatable MRI probes were incubated with $S$. aureus samples, whereas no change in intensity was observed for S. epidermidis and bacterial culture media (Fig. 5C). Moreover, a concentration-dependent effect in brightening from lower to higher concentrations of activatable MRI probes $(0,0.03$, and $0.07 \mathrm{Fe} \mathrm{mM})$ was also observed for $S$. aureus samples. Next, we calculated the signal-to-noise ratios (SNR) using the culture media as control (Fig. 5D). We have found 3.80- and 6.49-fold signal increases for $S$. aureus samples over $S$. epidermidis (non-targeted bacteria) when the activatable MRI probe was used at 0.03 and $0.07 \mathrm{mM}$ concentrations $(\mathrm{Fe})$, respectively. In contrast, $T_{2}$ relaxation times, the $r_{2}$ calculations, the MR images, and
Fig. 5 Specific detection of bacteria using 9.4-T MR imaging. (A) $T_{1}$ relaxation times for $S$. aureus (target bacteria), and controls: $S$. epidermidis (non-target bacteria) and culture media. (B) Bar graph of $R_{1}$ relaxation rate values for target bacteria and controls. (C) Contrast changes in $T_{1}$-weighted MR phantom imaging reflecting the activation of the MRI probe. (D) SNR values for S. aureus and S. epidermidis. Data are shown as mean $\pm \mathrm{SD}$ of $n=3$ experiments
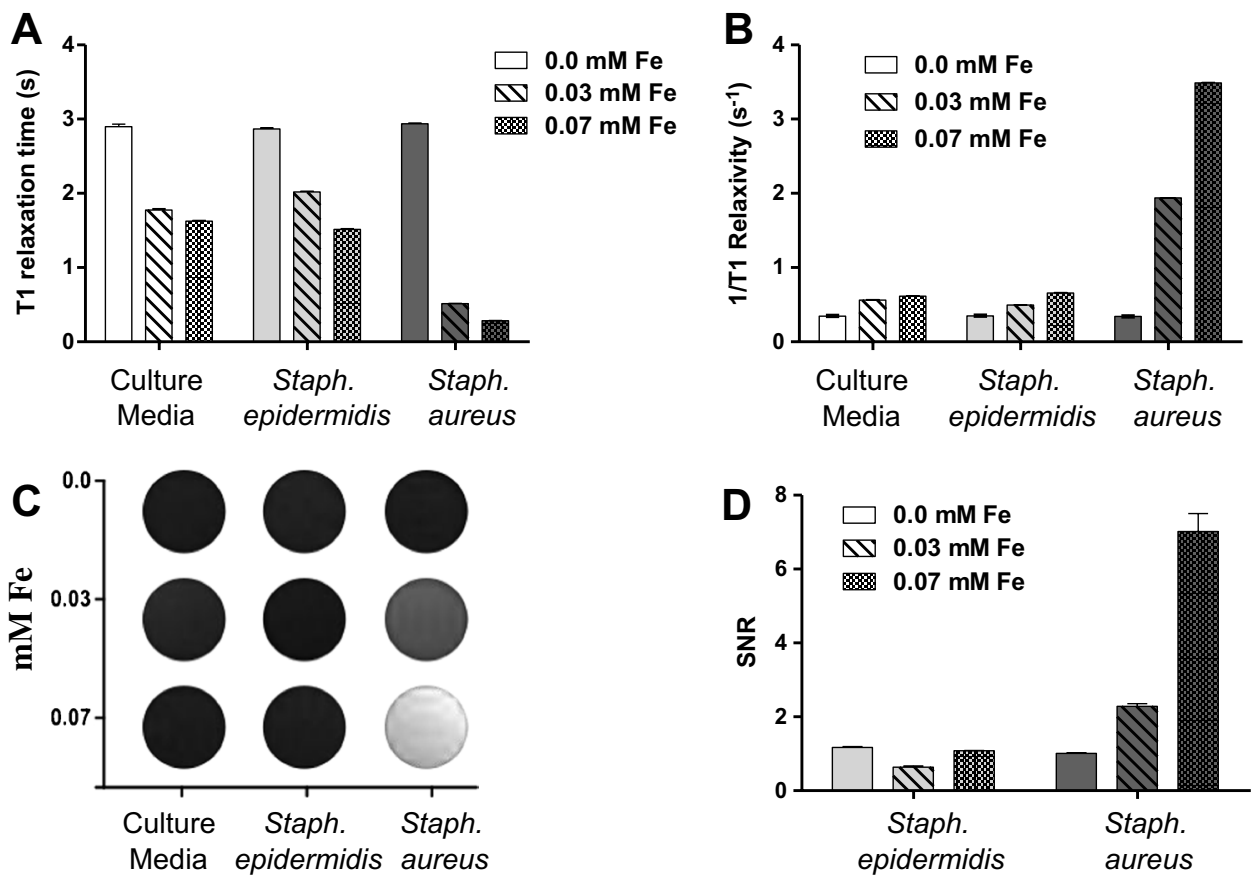
the $T_{2}$ SNR have shown limited capability to differentiate S. aureus from $S$. epidermidis (Fig S3). In addition, the $T_{2}$ SNR calculations have reported 1.47- and 1.70-fold signal increases for $S$. aureus samples over $S$. epidermidis using 0.03 and $0.07 \mathrm{mM}$ concentrations $(\mathrm{Fe})$, respectively. The comparative analysis of the $T_{1}$ and $T_{2}$ signals demonstrates that the activatable MRI probes reported in this study behave more efficiently as a $T_{1}$ rather than a $T_{2}$ contrast agent. All together, these results confirm the feasibility of activatable MRI probes for detecting biological markers such as nucleases derived from bacteria, demonstrating the potential of this approach for in vivo translation.

\section{Discussion}

The development of activatable MRI probes that respond to a biochemical stimulus has increased the attention of the scientific community in the last decade. Specific detection of biomarkers such as sugars, proteins, and enzymes has expended the diagnostic capabilities of MRI technologies by providing information at the cellular and molecular levels. These emerging activatable features, combined with the anatomical and functional imaging of standard MRI systems, can create alternative diagnostic strategies beyond the currently existing methods in the clinic.

This study reports, for the first time, on an activatable contrast agent with the capability to detect bacteria in a specific manner using MRI. The diagnosis of infectious diseases such as osteomyelitis, where $S$. aureus is one of the most prevalent pathogens, can be significantly improved by combining the specificity provided by our MRI probe and the unlimited tissue penetration features of the MRI technology. We envision that this approach could be potentially expanded to other bacteria or other human conditions, such as cancer.

Supplementary Information The online version contains supplementary material available at https://doi.org/10.1007/s00216-021-03710-z.

Author contribution All the authors contributed to the manuscript. All the authors have given approval to the final version of the manuscript.

Funding Open access funding provided by Linköping University. This research was supported by the Knut and Alice Wallenberg Foundation and The Swedish Government Strategic Research Area in Materials Science on Advanced Functional Materials at Linköping University (Faculty Grant SFO-Mat-LiU No. 2009-00971).

\section{Declarations}

Conflict of interest F.J. Hernandez and L.I. Hernandez are inventors in a patent that describes the use of MRI probe as contrast agents. The other authors have no relevant financial or non-financial interests to disclose.
Open Access This article is licensed under a Creative Commons Attribution 4.0 International License, which permits use, sharing, adaptation, distribution and reproduction in any medium or format, as long as you give appropriate credit to the original author(s) and the source, provide a link to the Creative Commons licence, and indicate if changes were made. The images or other third party material in this article are included in the article's Creative Commons licence, unless indicated otherwise in a credit line to the material. If material is not included in the article's Creative Commons licence and your intended use is not permitted by statutory regulation or exceeds the permitted use, you will need to obtain permission directly from the copyright holder. To view a copy of this licence, visit http://creativecommons.org/licenses/by/4.0/.

\section{References}

1. Lacivita E, Leopoldo M, Berardi F, Colabufo NA, Perrone R. Activatable fluorescent probes: a new concept in optical molecular imaging. Curr Med Chem. 2012;19(28):4731-41.

2. Li L, Shi W, Wu X, Li X, Ma H. In vivo tumor imaging by a gamma-glutamyl transpeptidase-activatable near-infrared fluorescent probe. Anal Bioanal Chem. 2018;410(26):6771-7.

3. Medintz IL, Clapp AR, Mattoussi H, Goldman ER, Fisher B, Mauro JM. Self-assembled nanoscale biosensors based on quantum dot FRET donors. Nat Mater. 2003;2(9):630-8.

4. Giannetti A, Tombelli S, Baldini F. Oligonucleotide optical switches for intracellular sensing. Anal Bioanal Chem. 2013;405(19):6181-96.

5. Tsai HY, Kim H, Massey M, Krause KD, Algar WR. Concentric FRET: a review of the emerging concept, theory, and applications. Methods Appl Fluoresc. 2019;7(4):042001.

6. Pehlivan ZS, Torabfam M, Kurt H, Ow-Yang C, Hildebrandt N, Yuce M. Aptamer and nanomaterial based FRET biosensors: a review on recent advances (2014-2019). Mikrochim Acta. 2019;186(8):563.

7. Rowland CE, Brown CW, Medintz IL, Delehanty JB. Intracellular FRET-based probes: a review. Methods Appl Fluoresc. 2015;3(4):042006.

8. Geissler D, Hildebrandt N. Recent developments in Forster resonance energy transfer (FRET) diagnostics using quantum dots. Anal Bioanal Chem. 2016;408(17):4475-83.

9. Pieper-Furst U, Kleuser U, Stocklein WF, Warsinke A, Scheller FW. Detection of subpicomolar concentrations of human matrix metalloproteinase-2 by an optical biosensor. Anal Biochem. 2004;332(1):160-7.

10. Ray PC, Fan Z, Crouch RA, Sinha SS, Pramanik A. Nanoscopic optical rulers beyond the FRET distance limit: fundamentals and applications. Chem Soc Rev. 2014;43(17):6370-404.

11. Sonnichsen C, Reinhard BM, Liphardt J, Alivisatos AP. A molecular ruler based on plasmon coupling of single gold and silver nanoparticles. Nat Biotechnol. 2005;23(6):741-5.

12. Antony PM, Trefois C, Stojanovic A, Baumuratov AS, Kozak K. Light microscopy applications in systems biology: opportunities and challenges. Cell Commun Signal. 2013;11(1):24.

13. Zhou C, Jiang M, Du J, Bai H, Shan G, Kwok RTK, et al. One stone, three birds: one AIEgen with three colors for fast differentiation of three pathogens. Chem Sci. 2020;11(18):4730-40.

14. He JX, Le Mai HK, Kho SH, Guo Z, Zhong W, Venkata Thappeta $\mathrm{KR}$, et al. Synthetic biohybrid peptidoglycan oligomers enable pan-bacteria-specific labeling and imaging: in vitro and in vivo. Chem Sci. 2020;11(12):3171-9.

15. Wang S, Zhou Z, Wang Z, Liu Y, Jacobson O, Shen Z, et al. Gadolinium metallofullerene-based activatable contrast agent for tumor signal amplification and monitoring of drug release. Small. 2019;15(16):1900691. 
16. Mi P, Kokuryo D, Cabral H, Wu H, Terada Y, Saga T, et al. A $\mathrm{pH}$-activatable nanoparticle with signal-amplification capabilities for non-invasive imaging of tumour malignancy. Nat Nanotechnol. 2016;11(8):724-30.

17. Kim T, Cho E-J, Chae Y, Kim M, Oh A, Jin J, et al. Urchin-shaped manganese oxide nanoparticles as $\mathrm{pH}$-responsive activatable $\mathrm{T} 1$ contrast agents for magnetic resonance imaging. Angew Chem Int Ed. 2011;50(45):10589-93.

18. Roda A, Michelini E, Caliceti C, Guardigli M, Mirasoli M, Simoni P. Advanced bioanalytics for precision medicine. Anal Bioanal Chem. 2018;410(3):669-77.

19. Santra S, Jativa SD, Kaittanis C, Normand G, Grimm J, Perez JM. Gadolinium-encapsulating iron oxide nanoprobe as activatable NMR/MRI contrast agent. ACS Nano. 2012;6(8):7281-94.

20. Hernandez FJ, Hernandez LI, inventorsAgent for use in the detection of nuclease activity. Patent WO2016062726A12014.

21. Choi JS, Kim S, Yoo D, Shin TH, Kim H, Gomes MD, et al. Distance-dependent magnetic resonance tuning as a versatile MRI sensing platform for biological targets. Nat Mater. 2017;16(5):537-42.

22. Shin TH, Kang S, Park S, Choi JS, Kim PK, Cheon J. A magnetic resonance tuning sensor for the MRI detection of biological targets. Nat Protoc. 2018;13(11):2664-84.

23. Choi JS, Lee JH, Shin TH, Song HT, Kim EY, Cheon J. Selfconfirming, "AND" logic nanoparticles for fault-free MRI. J Am Chem Soc. 2010;132(32):11015-7.

24. Zhen X, Lundborg CS, Zhang M, Sun X, Li Y, Hu X, et al. Clinical and economic impact of methicillin-resistant Staphylococcus aureus: a multicentre study in China. Sci Rep. 2020;10(1):3900

25. Kirwin E, Varughese M, Waldner D, Simmonds K, Joffe AM, Smith S. Comparing methods to estimate incremental inpatient costs and length of stay due to methicillin-resistant Staphylococcus aureus in Alberta, Canada. BMC Health Serv Res. 2019;19(1):743.

26. Yang W. Nucleases: diversity of structure, function and mechanism. Q Rev Biophys. 2011;44(1):1-93.

27. Hernandez FJ, Huang L, Olson ME, Powers KM, Hernandez LI, Meyerholz DK, et al. Noninvasive imaging of Staphylococcus aureus infections with a nuclease-activated probe. Nat Med. 2014;20(3):301-6.

28. Hongmu P, Marie-Christine D, editors. Studies of MRI relaxivities of gadolinium-labeled dendrons. ProcSPIE; 2011.

29. Gündüz S, Power A, Maier ME, Logothetis NK, Angelovski G. Synthesis and characterization of a biotinylated multivalent targeted contrast agent. ChemPlusChem. 2015;80(3):612-22.

30. Gündüz S, Savić T, Toljić Đ, Angelovski G. Preparation and in vitro characterization of dendrimer-based contrast agents for magnetic resonance imaging. J Vis Exp. 2016;118:54776.

31. Guo C, Hu J, Bains A, Pan D, Luo K, Li N, et al. The potential of peptide dendron functionalized and gadolinium loaded mesoporous silica nanoparticles as magnetic resonance imaging contrast agents. J Mater Chem B. 2016;4(13):2322-31.

32. Zhou G, Khan F, Dai Q, Sylvester JE, Kron SJ. Photocleavable peptide-oligonucleotide conjugates for protein kinase assays by MALDI-TOF MS. Mol Biosyst. 2012;8(9):2395-404.

33. Zhu X, Lin H, Wang L, Tang X, Ma L, Chen Z, et al. Activatable $\mathrm{T} 1$ relaxivity recovery nanoconjugates for kinetic and sensitive analysis of matrix metalloprotease 2. ACS Appl Mater Interfaces. 2017;9(26):21688-96.

34. Balian A, Garcia Gonzalez J, Bastida N, Akhtar KK, Borsa BA, Hernandez FJ. Kinetic screening of nuclease activity using nucleic acid probes. J Vis Exp. 2019(153).

35. Shin TH, Choi JS, Yun S, Kim IS, Song HT, Kim Y, et al. T(1) and T(2) dual-mode MRI contrast agent for enhancing accuracy by engineered nanomaterials. ACS Nano. 2014;8(4):3393-401.
36. Hernandez FJ, Hernandez LI, Kavruk M, Arica YM, Bayramoglu G, Borsa BA, et al. NanoKeepers: stimuli responsive nanocapsules for programmed specific targeting and drug delivery. Chem Commun (Camb). 2014;50(67):9489-92.

37. Cotin G, Blanco-Andujar C, Nguyen DV, Affolter C, Boutry S, Boos A, et al. Dendron based antifouling, MRI and magnetic hyperthermia properties of different shaped iron oxide nanoparticles. Nanotechnology. 2019;30(37):374002.

Publisher's Note Springer Nature remains neutral with regard to jurisdictional claims in published maps and institutional affiliations.

Prabu Periyathambi is a former member of the Nucleic Acids Technologies Laboratory (NAT-Lab); his main work was focused on the development of novel MRI probes using nucleic acids.

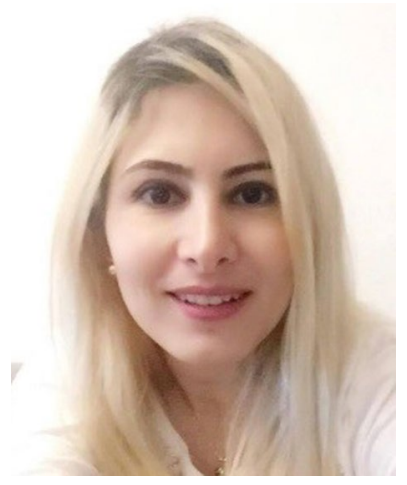

Alien Balian is a $\mathrm{PhD}$ student at the Nucleic Acid Technologies Laboratory (NAT-Lab) affiliated with the Department of Physics, Chemistry and Biology (IFM) under Frank J. Hernandez. She has worked with mammalian cell culture, cell toxicity assays, cell imaging, and MRI nucleic acid probe for non-invasive diagnosis of breast cancer. Before being affiliated at NAT-Lab, She has earned a masters degree in medical biology at Linköping University, and bachelor degree in pharmacology and pharmaceutical chemistry.

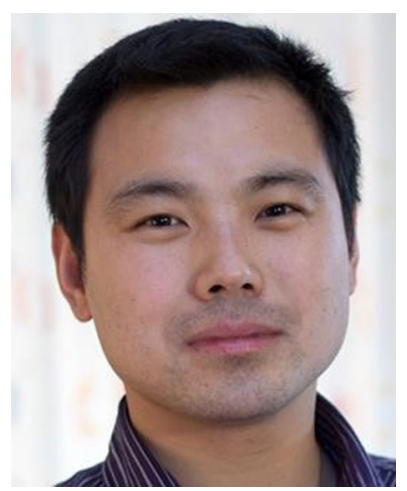

Zhangjun Hu is Senior Lecturer at Linköping University. His current research interests focus on molecules, complexes, and nanomaterials for biomedical imaging/sensing, and functional materials for new sustainable purposes, e.g., surface coating, lithium battery (LIBs), electrolysis of water, and perovskite OLED/solar cells.

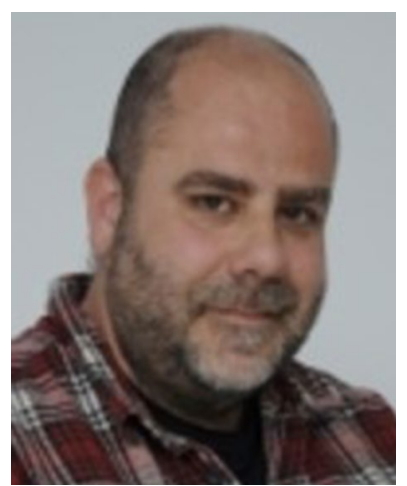

Daniel Padro is the NMR/MRI Platform Manager at CIC biomaGUNE, San Sebastian, Spain. He is mainly engaged in applications of magnetic resonance: nanoparticles, metabolomics, structural biology, heteronuclear applications to in vivo imaging. 


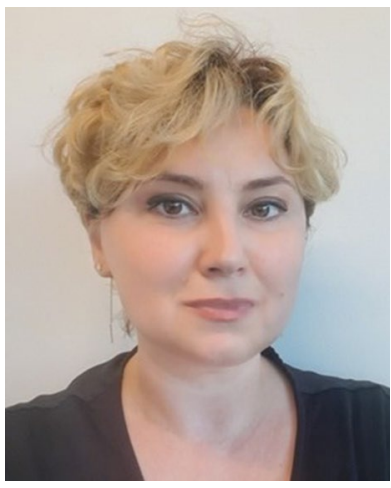

Luiza I. Hernandez is Chief Technology Officer and co-founder of SOMAprobes, San Sebastian, Spain and former Assistant Professor in the Department of Clinical and Experimental Medicine (IKE) at Linkoping University, Sweden. She has been working for over 10 years at the intersection between the fields of chemistry, biochemistry, cancer biology, microfluidics, RNA-based technologies for diagnostics and therapeutics. She is an expert in nucleases as biomarkers of disease (cancer and infectious diseases) and nuclease-based diagnostic technologies. She is currently leading SOMAprobes's research and development efforts to commercialize the nuclease-based technology applications.

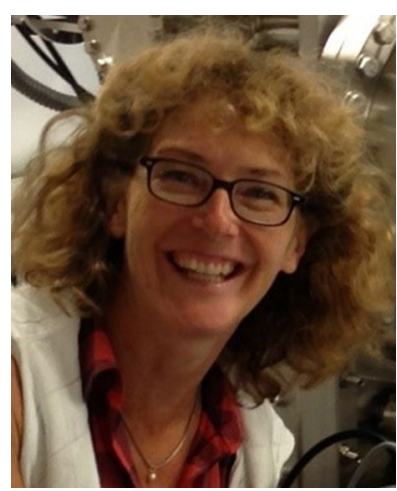

Kajsa Uvdal is Head of the Division Molecular Surface Physics and Nanoscience, and Director of CeNano at Linköping University, Sweden. She is a physicist by training and added biology to facilitate interdisciplinary research possibilities. She is focused on nanoprobes for biomedical imaging collaborating with Center of Medical Imaging and Visualization Faculty of Health, LiU. She also has long experience in surface spectroscopy and synchrotron-based research, at MaxIV National

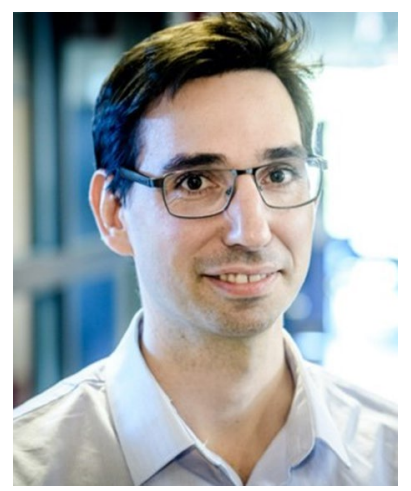

João M.N. Duarte is Head of the Diabetes and Brain Function lab at Lund University, and fellow of the Wallenberg Center for Molecular Medicine, Sweden. For many years, he has been using magnetic resonance tools to investigate metabolic pathways in living rodents, how brain energy metabolism is coupled to neuronal function, and on understanding the impact of metabolic disorders on brain function.

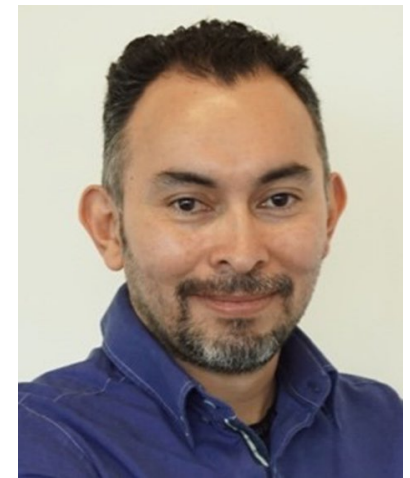

Frank J. Hernandez is Group Leader of the Nucleic Acids Technologies Laboratory, Senior Lecturer at Linköping University and Fellow of the Wallenberg Center for Molecular Medicine (WCMM) in Sweden. His lab is exploring the utility of nucleases as biomarkers of disease. His main research goal is to develop novel diagnostic and therapeutic strategies using nucleic acid probes for cancer and infectious diseases.

Synchrotron Laboratory, Lund, Sweden. 